\title{
Dengue: A Practical Experience of Medical Professionals in Hospital
} QUAZI TARIKUL ISLAM ${ }^{1}$, ARIFUL BASHER ${ }^{2}$, ROBED AMIN ${ }^{3}$

\begin{abstract}
:
Background: Dengue remains endemic and frequently intensifies into epidemics in Bangladesh since 2000 resulting in frequent hospitalizations.

Materials \& Methods: A prospective observational study was carried out to determine the clinical parameters of the subjects for hospitalization and the pattern of presentation of dengue fever in hospital care from July, 2010 to December, 2010.

Result: Total 50 cases were selected consecutively and diagnosed clinically as dengue, and were classified into 3 groups, i.e. 25 cases of classical dengue fever, 3 cases of dengue fever with unusual haemorrhage, 22 cases Dengue haemorrhagic fever (DHF-I and DHF-II) and all were discharged uneventfully. Among them 33(66\%) were male and outnumbered I $7(34 \%)$ were female. Mean ages of the subjects were $30.91 \pm 10.314,34.33 \pm$ 16.29 and $33.91 \pm 14.72$ years in respective groups. Majority had profound weakness, headache, myalgia, anorexia, nausea, and vomiting and itching. Diarrhoea, abdominal pain, organomegaly, ascites, and pleural effusion were not infrequent complaints of Dengue haemorrhagic fever patients, whereas occasional complaints in other groups. There is a linear relationship between platelet count and SGPT ( $p-0.037)$. Hametocrit value also markely increased in dengue haemorrhagic fever $(m-42.5)$ but not in dengue fever with unusual haemorrhage $(m-3 I)$. Biochemical marker specially Hct may be a good predictor to differentiate different presentation of dengue fever.
\end{abstract}

Conclusion: Patients with dengue syndrome showed varied presentation and the symptoms were nonspecific. So much attention has to be paid for early diagnosis and management.

Keyword: Dengue, Dengue haemorrhagic fever.

\section{Introduction:}

Dengue fever is found virtually throughout the tropics and cause an estimated 50-100 million illnesses annually, including 250,000-500,000 cases of dengue haemorrhagic fever (DHF) and 24,000 deaths. ${ }^{1,2}$ More than two fifths of the world's population ( 2.5 billion) lives in areas potentially at risk for dengue. $^{2}$ Dengue is considered to be one of the most important infectious diseases in these regions. ${ }^{3}$ The first documented outbreak of DF in Bangladesh was in 1965 when it was called "Dhaka fever". ${ }^{4}$ The aetiology was first determined when sero-epidemiological study of dengue infection was done at Chittagong Medical College hospital (CMCH), Chittagong in 1996 through 1997, and was found that $13.75 \%$ cases of fever were seropositive for dengue

1. Professor (PRL), Department of Medicine, Dhaka Medical College \& Hospital. Dhaka.

2. Junior Consultant, SK Hospital, Mymensingh

3. Assistant Professor, Department of Medicine, Dhaka Medical College \& Hospital. Dhaka.

Correspondence : Prof. Quazi Tarikul Islam, Professor (PRL), Department of Medicine, Dhaka Medical College \& Hospital. E-mail:prof.tarik@gmail.com infection. ${ }^{5}$ In Bangladesh, an outbreak of DF and DHF occurred in and around Dhaka city during the summer of 1999. Since then dengue cases are reported every year. Sporadic cases and small outbreaks went unreported until it took heavy toll in 2000 (5,555 cases and 93deaths); 2001 $(2,430$ cases and 44 deaths) and $2002(6,104$ cases and 58 death $)^{4,5}$. It is likely that the dengue epidemic will continue to increase in frequency and magnitude in future. ${ }^{6}$

Dengue Virus (DENV) infection leads to a verities of clinical manifestations that range from mild febrile illness as classical dengue fever (DF) to severe and fatal haemorrhagic disease as dengue haemorrhagic fever (DHF) and dengue shock syndrome (DSS). ${ }^{1}$ The risk of severe disease is much higher in sequential rather then primary infection. The etiologic agent (DENV) belongs to the Flavi viridae family and to the Flavivirus genus, with 4 serotypes. ${ }^{7}$ The physical examination in patient with $\mathrm{DF}$ is generally non specific. All entities initially present in a similar fashion with abrupt onset of fever, myalgia, arthralgia, rash and other non-specific features indistinguishable from one another. Hepatic dysfunction is common in dengue infection, and is attributed to a direct 
viral effect on liver cells or as a consequence of dysregulated host immune responses against the virus. ${ }^{7}$ The elevation of hepatic enzyme is usually modest (two to five times of upper normal value) but occasionally marked elevation was also noted. ${ }^{8}$ This report describes clinical, biochemical and serological studies of confirmed dengue infections at the hospital.

\section{Materials \& Methods:}

A prospective observational study was carried out to determine the clinical parameters of the subjects for hospitalization and the pattern of presentation of dengue fever in hospital care in Dhaka Medical College hospital and Popular Medical College Hospital from July, 2010 to December, 2010. Criteria for the diagnosis of dengue infection were based on clinical definition and the detection of IgM dengue $\mathrm{Ab}$ by ELISA method. DHF was differentiated from dengue fever (DF), and DHF was classified by disease severity as grades I, II, III and IV according to the national guidelines published by the DGHS GOB and WHO. ${ }^{2}$ Patients suffering from other chronic ailments in addition to dengue fever such as chronic renal failure, diabetes mellitus, skin infections, immunocompromised or haematological disorders are excluded from the study.

On admission, patients were carefully observed and pertinent clinical and laboratory data recorded daily on a standard record form. These included vital signs, petechiae or other skin haemorrhages, examination for signs of circulatory failure and presence of pleural effusion. X-ray examination of the chest were performed for cases whose showed physical findings corresponding to hydrothorax. Daily tourniquet tests were performed on all cases. Complete blood count(CBC), Haematocrit (Hct), platelet count and ALT (SGPT), AST(SGOT) were performed on all patients using blood samples obtained less than 24 hours after admission. Serial determinations of Hct and platelet counts were done daily or as frequently as every as needed. The platelet counts were done using the direct method (Rees and Ecker) with Wright's Giemsa stain. Aliquots of acute blood were tested for dengue (DEN) antibody, and blood samples were drawn for serology. The Chi square test with Yates' correction or Fisher's exact test was used to compare differences in the frequency of clinical manifestations among cases.

\section{Result:}

From July 01, 2010 to December 31, 2010, total 50 patients clinically suspected cases were confirmed as dengue infections. The age of the confirmed patients ranged from
16 years to 65 years, and the median age was 60 years. The male to female ratio was 1.94:1. Significant numbers of patients were student $10(20 \%)$ and doctors $10(20 \%)$ and others were from different professionals. On the basis of clinical and laboratory results all subject were categorized into three groups. Amongest, 25 cases of classical dengue fever, 3 cases of dengue fever with unusual hemorrhage, 22 cases hemorrhagic fever (DHF-I and DHF-II). The mean ages of the subjects were 30.91 $\pm 10.314,34.33 \pm 16.29$ and $33.91 \pm 14.72$ years in respective groups.

The most frequent findings were anorexia, retro orbital pain, red eye, rash, back pain, myalgia, and other constitutional syndrome. Table 1 shows the frequency of different symptoms and signs of different grade of dengue infection. Skin rashes occurred more frequently among patients with all groups of fever. Petechiae, positive tourniquet test and epistaxis were the most frequent hemorrhagic manifestations in DHF cases (Table-I). Pleural effusion and abdominal pain were found in one case of dengue fever with unusual haemorrhage and DHF grade III. All the patients had fever and almost all of them had temperature around $39^{\circ} \mathrm{C}$ and weakness. While continued fever was recorded in $52 \%$ patient, intermittent fever in $40 \%$ and remittent type was noticed in $8 \%$ of patients. Diarrhoea and abdominal pain were infrequent complains. Different types of rash i.e. macular, maculo-papular and erythematous rashes were observed in 34\% patients. Tourniquet test was positive in 22 (44\%) subjects. Organomegaly was observed very rarely. SGPT (ALT) was invariably raised in all patients with the duration of the course of the disease.

It has been observed that SGPT increased in all dengue patients irrespective of grade. But serum SGOT is raised more in DHF-II than other groups of dengue infection (fig-1). Of the 50 cases, platelet counts was done in $30(60 \%)$ patients and had thrombocytopenia in all cases. There is a significant difference among different grades. (P-0.037)(fig-2). There is a linear relationship between platelet count and serum SGPT (p-0.037) (fig-3). Patients with lower Platelet count having a higher SGPT. Haemoconcentration were observed in 30 cases. Haemetocrit (Hct) value also markely increased in dengue haemorrhagic fever (m- 42.5) but not in dengue fever with unusual haemorrhage (m-31) (fig-4). The majority (57.2\%) of the patients had a normal WBC $\left(5-10 \times 10^{3} / \mathrm{mm}^{3}\right)$. Leukopenia was recorded in 20 cases (40\%), and leukocytosis in 5 patients $(10 \%)$. 
Table-I

Signs and symptoms observed in 50 patients with confirmed dengue infections

\begin{tabular}{|c|c|c|c|c|c|}
\hline \multirow{2}{*}{$\begin{array}{l}\text { Finding } \\
\text { Finding }\end{array}$} & \multicolumn{4}{|c|}{ Classification } & \multirow[b]{2}{*}{$\begin{array}{c}\text { Total, } \\
\mathrm{N}=50(\%)\end{array}$} \\
\hline & $\begin{array}{c}\text { DF Classical } \\
\mathrm{N}=27,(\%)\end{array}$ & $\begin{array}{c}\text { DF with unsual } \\
\text { haemorrhage, } \mathrm{N}=3(\%)\end{array}$ & $\begin{array}{c}\text { DHF Grade I } \\
\mathrm{N}=8,(\%)\end{array}$ & $\begin{array}{c}\text { DHF Grade II, } \\
\mathrm{N}=12,(\%)\end{array}$ & \\
\hline Fever & $27(100 \%)$ & $3(100 \%)$ & $8(100 \%)$ & $12(100 \%)$ & $50(100 \%)$ \\
\hline Retro orbital pain & $15(55.55 \%)$ & $3(100.0 \%)$ & $4(50.0 \%)$ & $7(58.33 \%)$ & $29(58 \%)$ \\
\hline Eye redness & $11(40.74 \%)$ & $1(33.33 \%)$ & $1(12.5 \%)$ & $7(58.33 \%)$ & $20(40 \%)$ \\
\hline Rash & $8(29.63 \%)$ & $1(33.33 \%)$ & $4(50.0 \%)$ & $8(66.66 \%)$ & $21(42 \%)$ \\
\hline Chill & $9(33.33 \%)$ & $1(33.33 \%)$ & $7(87.5 \%)$ & $7(58.33 \%)$ & $24(48 \%)$ \\
\hline Back pain & 19(70.37\%) & $2(66.66 \%)$ & $6(75 \%)$ & $7(58.33 \%)$ & $34(68 \%)$ \\
\hline Joint pain & $15(55.55 \%)$ & $1(33.33 \%)$ & $4(50 \%)$ & $5(41.66 \%)$ & $25(50 \%)$ \\
\hline Myalgia & $19(70.37 \%)$ & $2(66.66 \%)$ & $6(75 \%)$ & $8(66.66 \%)$ & $35(70 \%)$ \\
\hline Neck pain & $6(22.22 \%)$ & $1(33.33 \%)$ & $4(50 \%)$ & $5(41.66 \%)$ & $16(32 \%)$ \\
\hline Anorexia, Nausea, Vomiting & $18(66.66 \%)$ & $3(100.0 \%)$ & $7(87.5 \%)$ & $5(41.66 \%)$ & $33(66 \%)$ \\
\hline Blood in stool & $1(3.70 \%)$ & $1(33.33 \%)$ & $0(0 \%)$ & $1(8.33 \%)$ & $3(6 \%)$ \\
\hline Nasal bleeding & $1(3.70 \%)$ & $1(33.33 \%)$ & $0(0 \%)$ & $0(0 \%)$ & $2(4 \%)$ \\
\hline Gum bleeding & $2(7.40 \%)$ & $1(33.33 \%)$ & $2(25 \%)$ & $3(25 \%)$ & $8(16 \%)$ \\
\hline Blood in urine & $0(0 \%)$ & $0(0 \%)$ & $0(0 \%)$ & $1(8.33 \%)$ & $1(2 \%)$ \\
\hline Haemoptysis & $0(0 \%)$ & $0(0 \%)$ & $1(12.5 \%)$ & $0(0 \%)$ & $1(2 \%)$ \\
\hline Vaginal bleeding & $1(3.70 \%)$ & $0(0 \%)$ & $0(0 \%)$ & $0(0 \%)$ & $1(2 \%)$ \\
\hline Less urinary output & $0(0 \%)$ & $1(33.33 \%)$ & $0(0 \%)$ & $0(0 \%)$ & $1(2 \%)$ \\
\hline Abdominal pain & $4(14.81 \%)$ & $2(66.66 \%)$ & $2(25 \%)$ & $1(8.33 \%)$ & $9(18 \%)$ \\
\hline Rash & $7(25.92 \%)$ & $1(33.33 \%)$ & $3(37.5 \%)$ & $6(50 \%)$ & $17(34 \%)$ \\
\hline Respiratory distress & $1(3.70 \%)$ & $0(0 \%)$ & $0(0 \%)$ & $0(0 \%)$ & $1(2 \%)$ \\
\hline Pallor & $1(3.70 \%)$ & $0(0 \%)$ & $1(12.5 \%)$ & $1(8.33 \%)$ & $3(6 \%)$ \\
\hline Diarrhoea & $2(7.40 \%)$ & $1(33.33 \%)$ & $0(0 \%)$ & $0(0 \%)$ & $3(6 \%)$ \\
\hline Headache & $1(3.70 \%)$ & $0(0 \%)$ & $0(0 \%)$ & $0(0 \%)$ & $1(2 \%)$ \\
\hline Ascites & $0(0 \%)$ & $1(33.33 \%)$ & $0(0 \%)$ & $0(0 \%)$ & $1(2 \%)$ \\
\hline Pleural effusion & $0(0 \%)$ & $0(0 \%)$ & $0(0 \%)$ & $1(8.33 \%)$ & $1(2 \%)$ \\
\hline
\end{tabular}

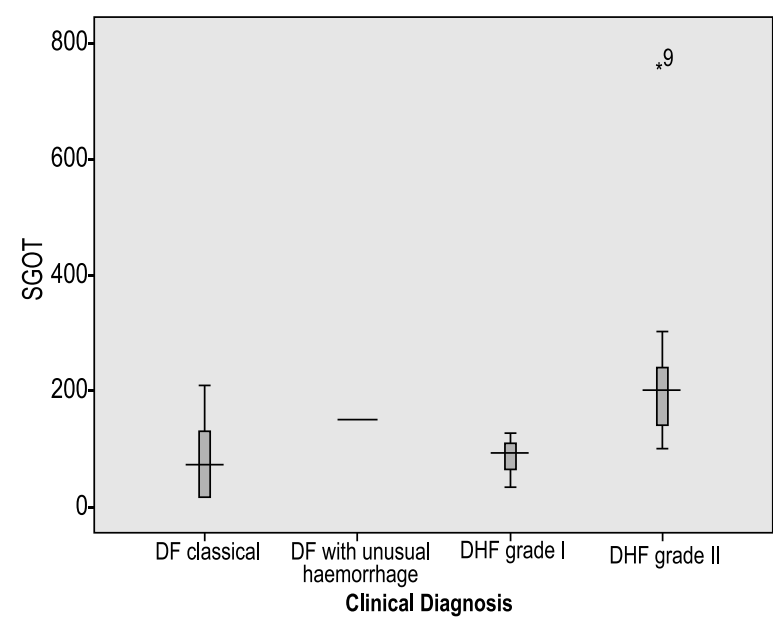

Fig-1: Box plot showing the label of SGOT among different grades of dengue infection.

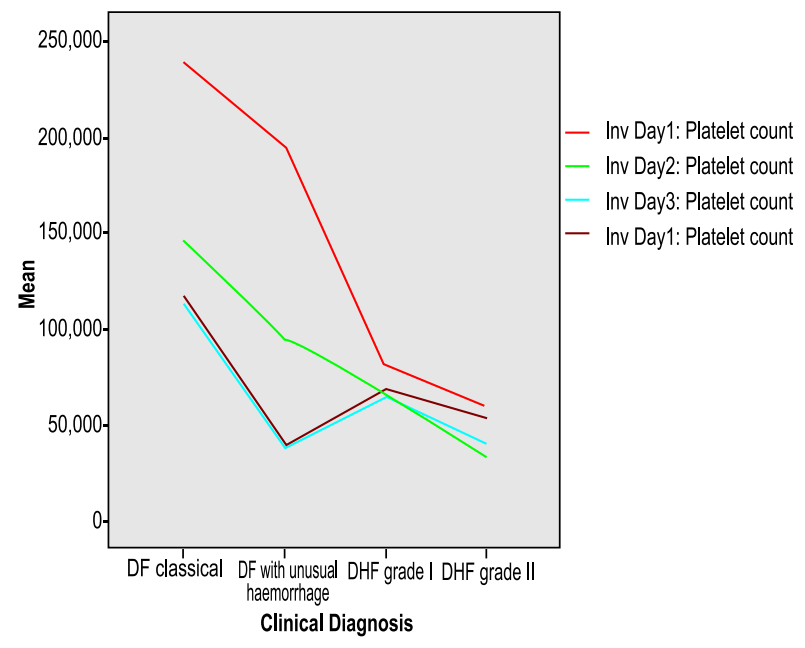

Fig-2: Platelet count among different groups of dengue infection 


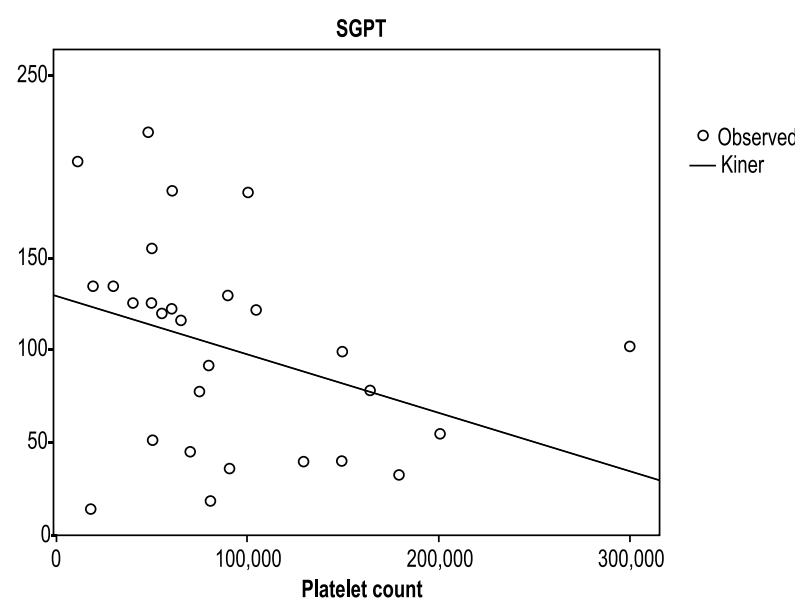

Fig-3: Relationship between platelet count and SGPT.

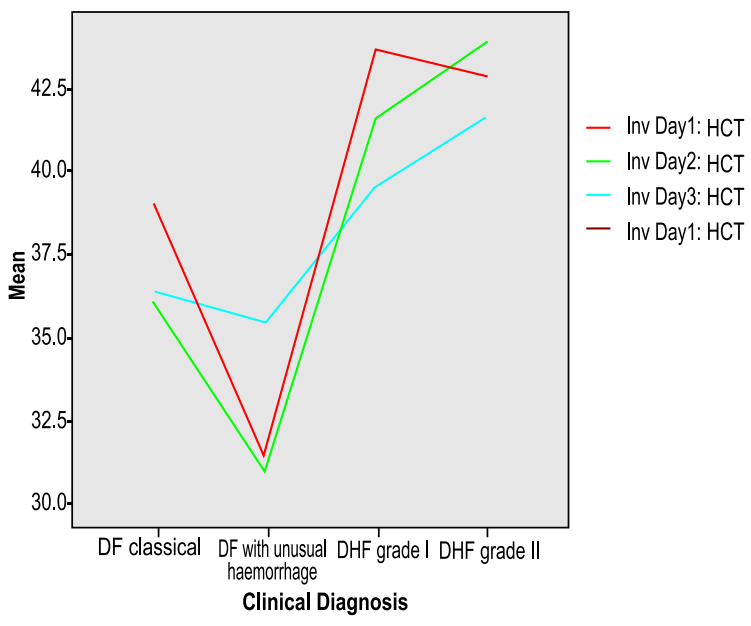

Fig-4: HCT level among different groups of dengue infection

\section{Discussion:}

The clinical findings are similar to those reported from other studies on confirmed dengue patients. Most of these patients developed mild hemorrhagic manifestations, but the occurrence of shock, particularly severe shock, was uncommon. This study shows that dengue infections continue to be a significant cause of hospitalization during periods of epidemic. Most of the current hospitalized cases are mild resulting in no case fatality rate from shock or massive haemorrhage. In this study, we primarily focused on the clinical manifestations and their associations with the biochemical and severity of 50 clinically and serologically proved dengue patients. We found different clinical features including fever (100\%), vomiting, headache, body ache, eye ache, itching, joint pain, loose motion, abdominal pain, anorexia (66\%), features of shock, gum bleeding (16\%), per vaginal bleeding $(2 \%)$, per rectal bleeding $(6 \%)$, hematemasis $(2 \%)$, hemoptysis $(2 \%)$, epistaxis $(4 \%)$, conjuntival congestion (40\%), sub conjunctival haemorrhage, relative bradycardia and low blood pressure(table-1). These features are consistent with typical cases of dengue fever but some percentage of different manifestations differ from studies conducted by Abu Bakar and Ahasan H A M N in khulna. ${ }^{9}$ In this study, 32\% patients had active bleeding of which gum bleeding was the commonest presentation. Studies depict some unpredictable sites of active bleeding like haematuria, per rectal and vaginal bleeding (table-1). Tourniquet test was positive in $22(44 \%)$ subjects. These findings are close to the study done by Arif KM et al. ${ }^{10}$ As we collected data from a hospital where treatment facilities were satisfactory and most of the patients came here without delay, marked complications or bleeding manifestation were less. Moreover, none of our patients died nor had any long term squeal. This improvement in survival rate in our study might be a reflection of awareness among people of Dengue in the recent years.

In this study, aminotransferases level was much higher in severely ill patients. These findings were similar to a study by Kabir A. ${ }^{11}$ AST and ALT are liver enzymes involved in amino acid metabolism. Hepatic dysfunction is common in dengue infection, and is attributed to a direct viral effect on liver cells or as a consequence of dysregulated host immune responses against the virus. ${ }^{12}$ Therefore, AST, ALT are valuable parameters for the evaluation of the severity of the disease. In a study carried out by Nguyen et al. ${ }^{13}$ and Uddin $\mathrm{KN}$ et al, ${ }^{14} \mathrm{AST}$ values were reported to be frequently abnormal, reaching values higher than those of ALT, we have found similar findings in our study. ${ }^{15}$ Therefore, AST, ALT are valuable parameters for the evaluation of the severity of the infection. 16

Plasma leakage usually occurs between three and seven days after the onset of illness. ${ }^{17}$ This coincides with severe thrombocytopenia and elevation of amino transferase. ${ }^{18}$ The study showed that there is a linear relationship between ALT and Platelet count (fig-3). ALT level is gradually increased when platelet count gradually lowered. The study also gives an idea about haematocrit and grading of dengue infection. Hct level are markedly decreased in dengue fever with unusual haemorrhage rather then other grade of dengue fever, even it is increased in grade two(fig-4). This finding may further evaluate the prediction of types of severity. However large scale study will be needed to establish this biochemical parameter as a severity tools.

The World Health Organization (WHO) previously classified symptomatic dengue virus infections into three categories for reporting purposes: undifferentiated fever, classic dengue fever, and dengue hemorrhagic fever (DHF). These categories have recently been controversial for several reasons. ${ }^{2}$ 
As a result of these problems, recently published guidelines from the WHO have adopted a revised classification of "dengue" and "severe dengue", where "severe dengue" is applied to patients who show severe plasma leakage (ie, leading to shock or fluid accumulation with respiratory distress), severe hemorrhage (as defined by the treating physician), or severe organ impairment (defined as aspartate transaminase (AST) or ALT e"1000, impaired consciousness, or severe involvement of the heart or other organs). ${ }^{19}$ Our study findings will also highlighting the value of new classification.

All the subjects in this study made an uneventful recovery with the supportive therapy, \& close monitoring only. Early diagnosis and prompt management of plasma leakage was the mainstay to overcome the menace. ${ }^{20}$

\section{Conclusion:}

In this study, we tried to find out the clinical parameters with varied presentations to create better awareness and clinically diagnostic skills among the health care providers and people to identify and refer the patients promptly to proper health care facilities to avert the ultimate danger. Liver enzyme invariably increase in all groups of Dengue fever and has got inverse relation to Platelet count. Therefore, the use of liver tests to evaluate the degree of liver damage is of great importance, and markers such as AST and ALT may be used as parameters to evaluate severity.

\section{Conflict of Interest : None}

\section{References:}

1. Gubler DJ. Dengue and dengue hemorrhagic fever. Clin Microbiol Rev 1998; 11: 480

2. World Health Organization. Dengue haemorrhagic fever: diagnosis, treatment, prevention and control, 2nd edn. Geneva: WHO, 1997

3. Parry J. Experts predict big rise in dengue fever in South East Asia. BMJ 2003; 327: 1368

4. Aziz MA, Graham RR, Gregg MB.” Dacca fever" An outbreak of dengue. Pak j Med Res 1967;6:83-92

5. World Health Organization. Dengue fever and dengue haemorrhagic fever. In: http://www.whoban.org/ communicable_dis_dengue.html. Accessed 26 January, 2009.

6. Hanif M, Devendra N S, Robed M A, BASHER A, AHMED T, Clinical Profile and Outcome of Patients with Dengue Syndrome In Hospital Care, J MEDICINE 2011; $12: 131-138$
7. Kabir A, Abdullah AA, Sadeka MM et al. The impact of dengue on liver function as evaluated by aminotransferase levels. J MEDICINE 2008; 9 : 66-68

8. Schwartz E, Mendelson E, Sidi Y, Dengue fever among travelers., Am J Med. 1996;101(5):516

9. Bakar A, Ahsan HAMN, Ahsan M, Mamun AA, Karim SR. Emergence of Dengue in Bangladesh. PAFMJ 2004;54(2):147-50

10. Arif KM, Mohammed FR, Nur Z et al. Clinical profile and outcome of dengue hemorrhagic fever in a tertiary care hospital in dhaka. J MEDICINE 2009;10:12-15

11. Kabir A, Abdullah AA, Sadeka MM. The Impact Of Dengue On Liver Function As Evaluated By Aminotransferase Levels. J MEDICINE 2008;9:66-68

12. Kalayanarooj S, Vaughn DW, Nimmannitya S, Green S, Suntayakorn S, Kunentrasai N, et al, Early clinical and laboratory indicators of acute dengue illness., J Infect Dis. 1997; 176(2):313

13. Nguyen TL, Nguyen TH, Tieu NT. The impact of dengue hemorrhagic fever on liver function. Res Virol 1997; 148: 273-7

14. Uddin KN, Musa AKM, Haque WMM, et al. A follow up on biochemical parameters in dengue patients attending BIRDEM hospital. Ibrahim Med. Coll. J. 2008; 2(1): $25-27$

15. Guzman MG, Kouri GP, Bravo J, Soler M, Vazquez S, Santos M, Villaescusa R, Basanta P, Indan G, Ballester JM,Dengue haemorrhagic fever in Cuba. II. Clinical investigations., Trans R Soc Trop Med Hyg. 1984;78(2):239

16. Wilder-Smith A, Tambyah PA, Severe dengue virus infection in travelers, J Infect Dis. 2007; 195(8): 1081

17. Anderson KB, Chunsuttiwat S, Nisalak A, Mammen MP, Libraty DH et al. Burden of symptomatic dengue infection in children at primary school in Thailand: a prospective study, Lancet. 2007; 369(9571): 1452

18. Schatzmayr HG, Nogueira RM, Travassos da Rosa,. An outbreak of Dengue Virus at Rio de Janeiro- 1986. Mem Inst Oswaldo Cruz 1986; 81: 245-6

19. Anonymous. Dengue: guidelines for diagnosis, treatment, prevention and control- new edition. World Health Organization, Geneva 2009, p. 1

20. National Guideline on Dengue and DHF $2^{\text {nd }}$ Edition, 2010, DGHS, MOH GOB 